\title{
The Seroprevalence of Human Cystic Echinococcosis in Iran: A Systematic Review and Meta-Analysis Study
}

\author{
Reza Shafiei, ${ }^{1}$ Saeed Hosseini Teshnizi, ${ }^{2}$ Kurosh Kalantar, ${ }^{3}$ \\ Maryam Gholami, ${ }^{1}$ Golnush Mirzaee, ${ }^{4}$ and Fatemeh Mirzaee ${ }^{5}$ \\ ${ }^{1}$ Vector-Borne Diseases Research Center, North Khorasan University of Medical Sciences, Bojnurd, Iran \\ ${ }^{2}$ Clinical Research Development Center of Children Hospital, Hormozgan University of Medical Sciences, Bandar Abbas, Iran \\ ${ }^{3}$ Department of Immunology, School of Medicine, Shiraz University of Medical Sciences, Shiraz, Iran \\ ${ }^{4}$ Rehabilitation Management, University of Social Welfare and Rehabilitation Sciences, Tehran, Iran \\ ${ }^{5}$ School of Nursing and Midwifery, Shahrekord University of Medical Sciences, Shahrekord, Iran
}

Correspondence should be addressed to Saeed Hosseini Teshnizi; saeed.teshnizi@gmail.com

Received 6 June 2016; Revised 11 August 2016; Accepted 28 August 2016

Academic Editor: José F. Silveira

Copyright (C) 2016 Reza Shafiei et al. This is an open access article distributed under the Creative Commons Attribution License, which permits unrestricted use, distribution, and reproduction in any medium, provided the original work is properly cited.

\begin{abstract}
Human cystic echinococcosis (HCE), a zoonotic infection of the larval stage of Echinococcus granulosus, has high effect on public health in human population all around the world. Iran is one of the most important endemic areas in the Middle East. This systematic review and meta-analysis was performed to evaluate the seroprevalence of HCE in Iranian population. An electronic search for articles from 1985 until April 2015 was performed using data bases PubMed, Scopus, Google Scholar, Magiran, IranMedex, Iran Doc, and Scientific Information Database (SID) both in English and in Persian. A random-effects meta-analysis was used to combine results from individual studies. The information was analyzed by STATA version 11.1. A total of 33 articles met our eligibility criteria and were included in a meta-analysis. The pooled estimate of the prevalence of HCE based on random-effects model was estimated $6.0 \%$ (95\% CI: $4.0 \%, 7.0 \%$ ). The prevalence of the disease significantly increased with age and prevalence rate in males was significantly lower than females $(p<0.001)$. The using of CIE or CCIEP method was also significantly greater than the other methods $(p<0.001)$. There was a publication bias in prevalence of studies. HCE is highly prevalent in Iran. Public education for preventive strategies and finally reducing transmission of the parasite and infection in population is needed.
\end{abstract}

\section{Introduction}

Human hydatidosis or human cystic echinococcosis (HCE) is a chronic parasitic infection disease caused by the larval stage of Echinococcus granulosus which has an important effect on public health in human populations $[1,2]$. This zoonotic disease is initiated by accidental ingestion of the parasite's egg. It can be transmitted by infected feces of dogs via soil, vegetables, contact with dog, water, food, and so forth $[3,4]$.

The disease was widely distributed mostly in regions where sheep-rearing is a major industry $[5,6]$. This multihost disease is one of the most important public health infection diseases in Iran [6, 7]. Echinococcosis is one problem not only in humans but also in traps. It causes a huge economic burden for governments. That is why Ministry of Health and veterinary organizations pay more attention to it every year and have regular instruction programs for prevention of this infection. For example, they kill wild dogs; they also prescribe antihelminthes drugs in pet dogs and train the people to wash vegetables. The prevalence of hydatidosis in dogs has been recorded to be $5-45 \%$ in Iran [8]. The infection rate with different strains of E. granulosus sensu lato in various domestic livestock has been reported to be $24.41 \%, 8.51 \%, 18.89 \%$, $35.76 \%$, and $35.21 \%$ in sheep, goat, cattle, buffalo, and camels, respectively [8-10]. The incidence of surgical cases of HCE is estimated to be $1.18-3$ per 100,000 in different medical centers of Iran provinces and territories [3].

Early diagnosis of HCE is difficult due to being asymptomatic in early stages while using physical imaging; particularly ultrasound (US) examination is helpful not only 
at late stages but also for early diagnosis (cysts under $1 \mathrm{~cm}$ in diameter) $[2,11]$. The early diagnosis of HCE is based on available immunodiagnostic techniques with specific immunodominant antigens such as Ag B and US imaging. Methods for detecting specific antibodies can provide opportunities for early treatment of the disease [11-13]. Immunodiagnostic techniques have been used for total screening of population in endemic regions but the sensitivity and specificity of the diagnostic antigen are important [11, 12]. Several immunochemical tests such as ELISA and IFA are developed for determining anti-echinococcus IgG in serum for the diagnosis of HCE in Iran.

Given the high prevalence and the greater importance of this parasite, as well as the economic losses and the significant mortality and morbidity that HCE has in Iran, and due to the fact that the prevalence of this disease has not been identified in Iran yet, the need for a comprehensive study with the aim of serological monitoring of this disease in Iran seemed necessary. In this systematic review and metaanalysis study, we provide some insights into seroprevalence of HCE in different provinces of Iran from 1985 to 2015 where noncoordinated mass screenings have been performed in the past.

\section{Methods}

2.1. Search Method. The medical publications in English and Persian electronic databases were searched including PubMed Medical Subject Headings ( $\mathrm{MeSH} / \mathrm{mh}$ ), Google Scholar, Magiran, Iran Medex, Iran Doc, and Scientific Information Database (SID) both English and Persian databases from 1985 to April 2015. Publication searches were applied using the keywords: "Seroprevalence", "Serological prevalence", "Cystic Echinococcosis", "Hydatid cyst", "Hydatidosis", "Human", "IgG antibody", and "Iran" in combination or alone. To reduce the possibility of selection bias in this study, criteria were clearly defined and studied.

2.2. Data Extraction. The information was extracted from the included studies using a standard form by the two independently reviewers (RSH, STH). Any disagreement was resolved by discussion between the two reviewers. If consensus could not be reached, a third reviewer was consulted (MGH). Kappa index showed an agreement of $89 \%$ between the two reviewers.

The standard form consisted of the following variables: first author; year of publication; location, sample size, positive subjects, age and sex of participants, and lab methods. The outcome was the prevalence of seroprevalence and this was obtained for each study by dividing the number of positive cases to the total sample size.

2.3. Statistical Analysis. In this meta-analysis study outcomes were the prevalence of the seroprevalence of HCE. Forest plot was used to visualize the heterogeneity among studies. The results for each study and pooled outcome were revealed as a forest plot [reported as effect size (ES) with a $95 \%$ confidence interval (CI)]. Cochran's heterogeneity statistic or Q-test ( $p<0.1$ indicated heterogeneity) and $I^{2}$ statistic were used to examine the difference in study variability due

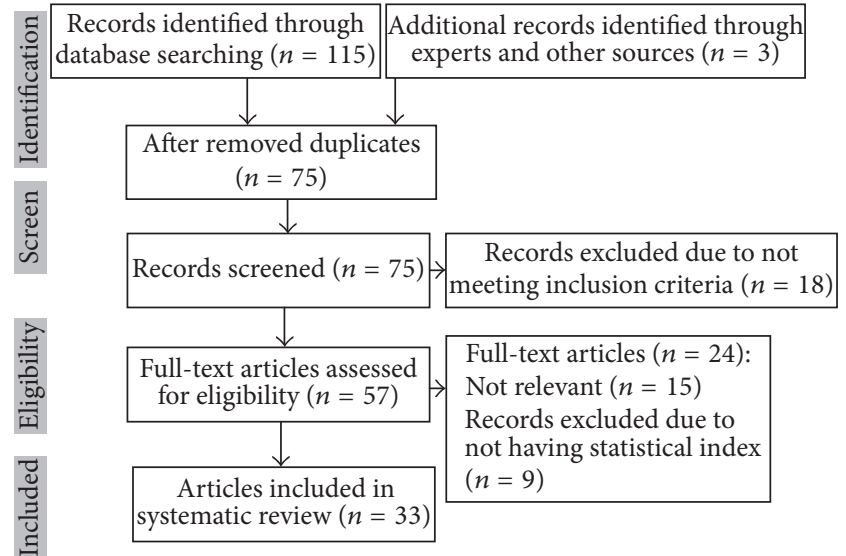

FIGURE 1: PRISMA flowchart describing the study design process.

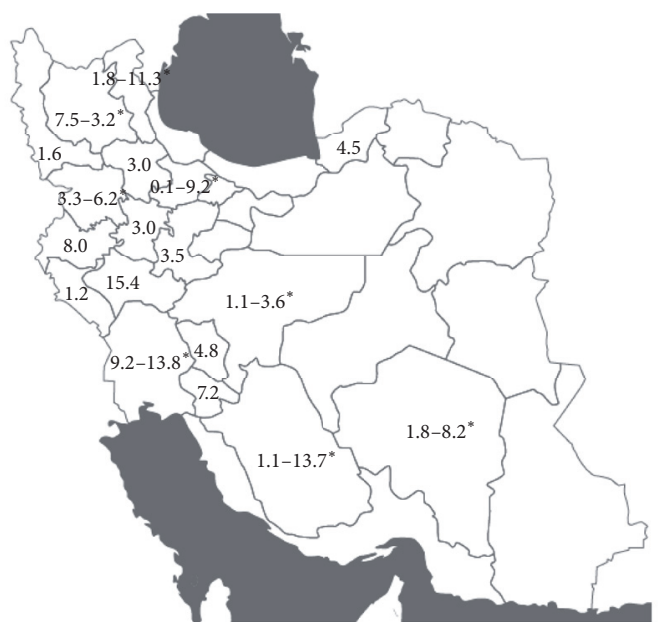

FIGURE 2: Graphic representation of prevalence (\%) of HCE in provinces in Iran. The asterisk $(*)$ means the minimum and maximum of prevalence in province.

to heterogeneity rather than chance, with a range from 0 to 100 percent (values of $25 \%, 50 \%$, and $75 \%$ are considered to represent low, medium, and high heterogeneity, resp.). Subgroup meta-analysis analysis was used to compare the prevalence of hydatidosis among age, sex, and lab methods groups. Egger's test was used to evaluate the publication bias $[14,15]$. Statistical analyses were conducted using the Statistical Software Package (STATA) version 11.1.

\section{Results}

Our initial database searches identified 115 articles and an additional 3 studies through hand searches and expert suggestions, giving a total of 118 articles that were screened. Out of these, 57 were chosen for reading of full text and 33 were included in this meta-analysis. Figure 1 shows the diagram of article selection according to the PRISMA statement.

Generally, the most prevalence of this disease took place in the western and southwestern areas of Iran and the highest prevalence was related to Lorestan, Fars, and Khuzestan provinces and the lowest rate was related to Tehran (Figure 2). 
TABLE 1: General characteristics of studies included in the present systematic review and meta-analysis.

\begin{tabular}{|c|c|c|c|c|c|c|c|}
\hline Author [reference] & Year & Province & sample & $\begin{array}{c}\text { Positive } \\
(n)\end{array}$ & $\begin{array}{c}\text { Positive } \\
\%(95 \% \text { CI })\end{array}$ & $\begin{array}{l}\text { Lab method } \\
(\mathrm{Ag})\end{array}$ & Target group \\
\hline Jamali [37] & 1995 & Uromieh & 300 & 5 & $1.67(0.22,3.12)$ & IFA & Villagers \\
\hline Arbabi $[18]$ & 1998 & Hamedan & 1530 & 46 & $3.01(2.15,3.86)$ & IFA & Healthy volunteers \\
\hline Saberi-Firouzi [21] & 1998 & Fars & 1000 & 137 & $13.70(11.57,15.83)$ & CIE & Healthy volunteers \\
\hline Mohamadi [38] & 1998 & Tehran & 700 & 68 & $9.71(7.52,11.91)$ & IFA & Healthy volunteers \\
\hline Zariffard [39] & 1999 & Western Iran & 4138 & 230 & $5.56(4.86,6.26)$ & ELISA & Healthy volunteers \\
\hline Nilfroshan [40] & 1998 & Esfehan & 1000 & 36 & $3.60(2.45,4.75)$ & IFA & Healthy volunteers \\
\hline Sadjjadi [13] & 2001 & Shiraz & 1227 & 76 & $6.19(4.85,7.54)$ & CCIEP & Healthy volunteers \\
\hline Sedaghat Gohar [27] & 2001 & Tehran & 1052 & 62 & $5.89(4.47,7.32)$ & IFA & Healthy volunteers \\
\hline Yousefi Darani [28] & 2003 & Chaharmahal & 2524 & 120 & $4.75(3.92,5.58)$ & CIE & Surgical patients \\
\hline Amiri [41] & 2001 & Kermanshah & 1072 & 86 & $8.02(6.4,9.65)$ & IFA & Patients and blood donors \\
\hline Farrokhzad [17] & 2004 & Tehran & 437 & 1 & $0.23(-0.22,0.68)$ & IFA & Healthy volunteers \\
\hline Haniloo [29] & 2002 & Zanjan & 2367 & 71 & $3.00(2.31,3.69)$ & ELISA & Healthy volunteers \\
\hline Aflaki [22] & 2005 & Eilam & 3000 & 37 & $1.23(0.84,1.63)$ & Dot-ELISA & Healthy volunteers \\
\hline Rafiei [42] & 2005 & Khuzestan & 4596 & 437 & $9.51(8.66,10.36)$ & ELISA & Healthy volunteers \\
\hline Akhlaghi [43] & 2005 & Kordestan & 1114 & 37 & $3.32(2.27,4.37)$ & IFA & Healthy volunteers \\
\hline Rafiei [20] & 2007 & Khuzestan & 3446 & 475 & $13.78(12.63,14.94)$ & ELISA & Healthy volunteers \\
\hline Baharsefat [34] & 2007 & Golestan & 1024 & 46 & $4.49(3.22,5.76)$ & ELISA, IFA & Healthy volunteers \\
\hline Mirzanejadasl [30] & 2008 & Ardabil & 1003 & 111 & $11.07(9.13,13.01)$ & ELISA & Healthy volunteers \\
\hline Hadadian [23] & 2008 & Kordestan & 1979 & 22 & $1.11(0.65,1.57)$ & ELISA & Healthy volunteers \\
\hline Moazezi [31] & 2009 & Kerman & 451 & 37 & $8.20(5.67,10.74)$ & ELISA & Blood donors \\
\hline Akhlaghi [44] & 2009 & Tehran & 1100 & 18 & $1.64(0.01,0.02)$ & Dot-ELISA & Healthy volunteers \\
\hline Esmaeili [45] & 2010 & Kashan & 361 & 11 & $3.05(0.01,0.05)$ & ELISA, IFA & Healthy volunteers \\
\hline Srakari [24] & 2010 & Yasuj & 500 & 36 & $7.20(0.05,0.09)$ & ELISA & Patients referred to lab \\
\hline Dadkhah [46] & 2011 & East Azarbaijan & 250 & 8 & $3.20(0.01,0.05)$ & IFA & Healthy volunteers \\
\hline Harandi [35] & 2011 & Kerman & 1140 & 34 & $2.98(0.02,0.04)$ & ELISA & Healthy volunteers \\
\hline Kavous [25] & 2010 & Jahrom & 1096 & 69 & $6.30(0.05,0.08)$ & ELISA & Patients referred to lab \\
\hline Garedaghi [47] & 2011 & East Azarbaijan & 1500 & 11 & $0.73(0,0.01)$ & ELISA & Healthy volunteers \\
\hline Heidari [26] & 2011 & Ardabil & 670 & 12 & $1.79(0.01,0.03)$ & ELISA & Healthy volunteers \\
\hline Rakhshanpour [36] & 2012 & Qom & 1564 & 25 & $1.60(0.01,0.02)$ & ELISA & Healthy volunteers \\
\hline Zibaei [16] & 2013 & Khorram abad & 617 & 95 & $15.40(0.13,0.18)$ & ELISA & Patients referred to lab \\
\hline Asgari [19] & 2013 & Arak & 578 & 20 & $3.46(0.02,0.05)$ & ELISA & Healthy volunteers \\
\hline Shahrokhabadi [48] & 2014 & Kerman & 486 & 9 & $1.85(0.01,0.03)$ & ELISA & Patients referred to HC \\
\hline Ilbeigi [33] & 2015 & Isfahan & 635 & 7 & $1.10(0,0.02)$ & ELISA & Patients referred to $\mathrm{HC}$ \\
\hline
\end{tabular}

$N$ : number of positive; HC: health center.

From 42706 people 2551 were positive for anti-echinococcosis. Indirect fluorescent antibody (IFA), enzyme-linked immunosorbent assay (ELISA), counterimmunoelectrophoresis (CIE) or counter-current immunoelectrophoresis (CCIEP) were used in all of the studies. ELISA was the mostly used test in this study, 17 (51.52\%). Target group of most of studies was healthy volunteers 24 (72.72\%) (Table 1).

There was a strong heterogeneity in the prevalence of the studies $\left(I^{2}=98 \%, p<0.0001\right)$. The pooled estimate of the prevalence of HCE based on random-effects meta-analysis was obtained $5.0 \%$ (95\% CI: 4.0\%, 6.0\%). The highest prevalence was related to study of Zibaei et al. (2013) with prevalence $15.4 \%$ in Khorramabad province [16] study carried out by ELISA and the lowest prevalence was $0.2 \%$ in Farrokhzad et al. (2004) in Tehran study carried out by IFA [17]. Also the pooled prevalence significantly was higher than zero line (ES $=0: z=10.03, p<0.001$ ) (Figure 3).

The subgroup analysis indicated that the prevalence of HCE was significantly increased with the increase of age $(p<$ 0.001). Also prevalence of CE among males, 2.1 (95\% CI: $1.8 \%$, $2.4 \%)$, was significantly lower than females, $3.6 \%$ (95\% CI: $3.2 \%, 3.9 \%)(p<0.001)$. Also the prevalence for CIE method 


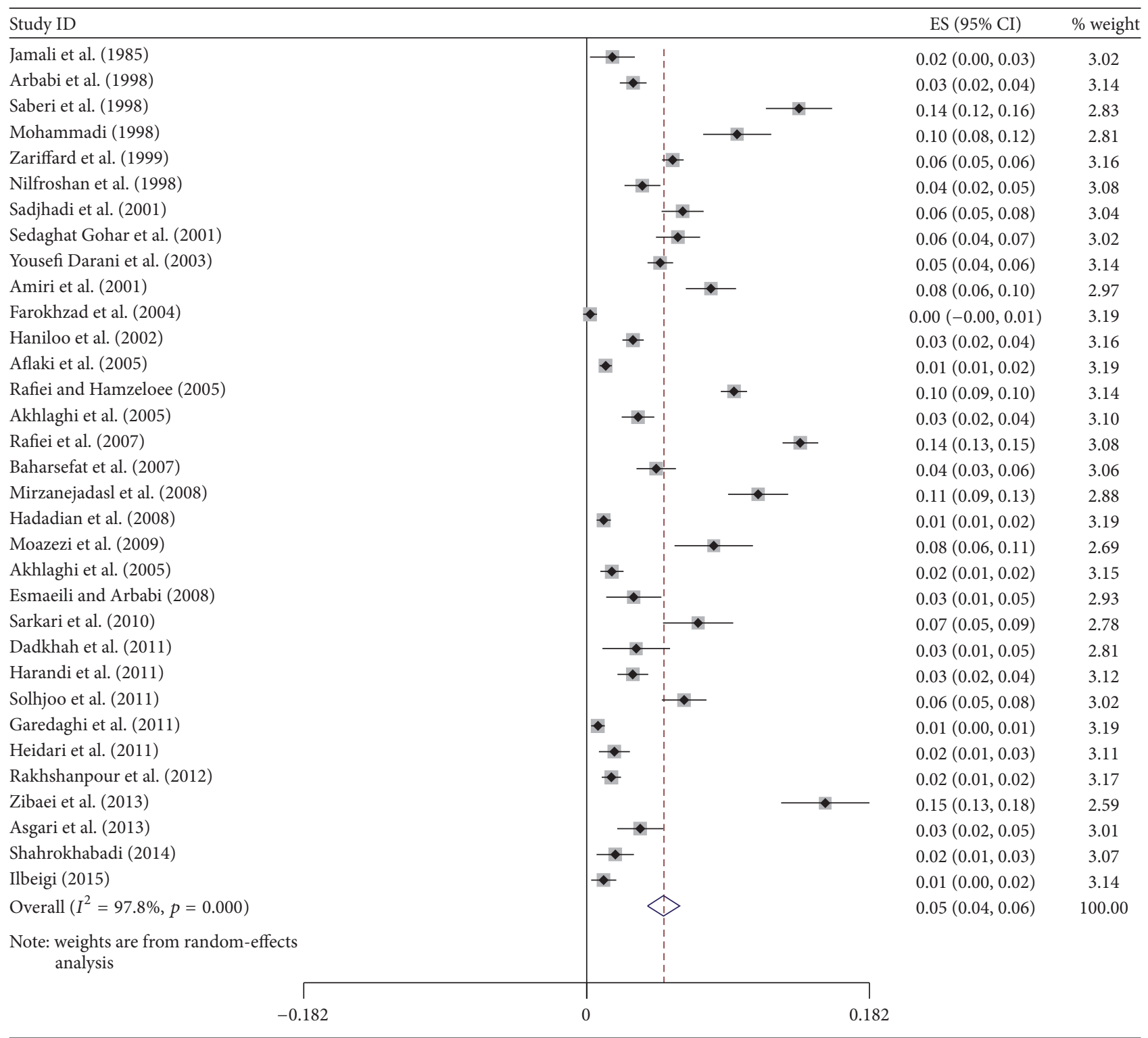

FIGURE 3: Forest plot of meta-analysis results for prevalence of CE. The middle point of each line indicates the prevalence rate and the length of line indicates $95 \%$ confidence interval of each study. Rhombus shape indicates $95 \%$ confidence interval for all studies.

was significantly greater than other methods $(p<0.001)$ (Table 2). The results of Egger's test showed there was not a publication bias among studies (coef. $=-0.011$, $\mathrm{SE}=0.008$, $p=0.326$ ). Therefore, this meta-analysis included two types of studies, high and low prevalence of HCE.

\section{Discussion}

HCE as an emerging neglected disease is a major public health problem in many countries which results in substantial economic resource loss [5]. The mentioned disease has a global distribution with an annual occurrence ranging from 1 to 200 per 100,000 individuals [1]. The prevalence rate of HCE based on hospital cases is different in Iran with rate of $>1 \%$ of total population $[7,18]$ and this is the most commonly used index of HCE [16]. Most of the infected people with the larva of E. granulosus have a delay in showing related symptoms such as cyst-like mass which grows gradually among various groups [5]. So serological examination alone is useful for giving an approximate evaluation of the infection pressure and might be useful (used on already collected serum samples, such as those found in blood banks) to provide data on the level of presence of E. granulosus in a given area to identify asymptomatic cyst carriers generally. We must also have plans to control this disease. To achieve a more accurate diagnosis, mass screening should include both ultrasound examination and serology [19].

The result of our investigation showed that the range of HCE is $0.2 \%$ in Tehran with IFA by Farrokhzad et al. (2004) [17] while Zibaei et al. recorded a higher seroprevalence 
TABLE 2: Subgroup meta-analysis of cystic echinococcosis for age, sex, and lab methods.

\begin{tabular}{|c|c|c|c|c|c|c|c|}
\hline \multirow{2}{*}{ Variable } & \multirow{2}{*}{ Number } & \multirow{2}{*}{$\begin{array}{c}\text { Prevalence } \\
(\%)\end{array}$} & \multicolumn{2}{|c|}{$95 \% \mathrm{CI}$} & \multirow{2}{*}{$I^{2}$} & \multirow{2}{*}{ Q-test } & \multirow{2}{*}{$p$} \\
\hline & & & Lower & Upper & & & \\
\hline \multicolumn{8}{|l|}{ Age (year) } \\
\hline$<30$ & 7 & 2.2 & 1.9 & 3.3 & $93.2 \%$ & \multirow{3}{*}{862.8} & \multirow{3}{*}{$p<0.001$} \\
\hline $30-40$ & 9 & 2.8 & 2.1 & 4.2 & $94.5 \%$ & & \\
\hline$>40$ & 6 & 4.1 & 3.0 & 4.8 & $98.7 \%$ & & \\
\hline \multicolumn{8}{|l|}{ Sex } \\
\hline Male & 21 & 2.1 & 1.8 & 2.4 & $94.9 \%$ & \multirow{2}{*}{398.1} & \multirow{2}{*}{$p<0.001$} \\
\hline Female & 10 & 3.6 & 3.2 & 3.9 & $96.2 \%$ & & \\
\hline \multicolumn{8}{|l|}{ Lab methods } \\
\hline CIE & 3 & 6.0 & 5.3 & 6.7 & $96.6 \%$ & \multirow{4}{*}{720.9} & \multirow{4}{*}{$p<0.001$} \\
\hline ELISA & 15 & 3.2 & 3.0 & 3.4 & $98.6 \%$ & & \\
\hline IFA & 9 & 2.2 & 1.8 & 2.5 & $97.4 \%$ & & \\
\hline ELISA \& Others & 4 & 1.8 & 1.5 & 2.1 & $91.8 \%$ & & \\
\hline
\end{tabular}

(15.4\%) in Khorramabad in the southwest of Iran with ELISA [16]. The prevalence of HCE infection in this survey was higher in southwest and south of Iran with $13.7 \%$ by Rafiei et al. (2007) in Khouzestan [20] and Saberi-Firouzi et al. (1998) in Fars [21]. The higher prevalence found by the studies which used counterimmunoelectrophoresis (CIE) or counter-current immunoelectrophoresis (CCIEP) may seem paradoxical since this test is usually considered to be more specific than sensitive (it is used as a confirmation test in some countries; it is time-consuming and is rarely used as a screening test) [18]. Besides this test, ELISA with B antigen was used in most of the studies (20 studies) because this method is acceptable, easy, efficient, and affordable and has a high level of sensitivity and specificity. In addition, since preparing the $\mathrm{B}$ antigen is easy, using local area antigen shows highly accurate test results. Applying of different kinds of serological methods could be the reason for controversy of obtained results. In addition, it seems that using the serological methods with high sensitivity and specificity would be helpful and something must be noticed that for each experiment in each part of world it is highly recommended to use the antigen which is prepared from that area. Thus, using the antigen which is from a specific district should be used in ELISA test. This strategy could have made the ELISA test more reliable in comparison to those tests which are using the universal antigen. So, we recommend that ELISA test by means of local antigens could help us to get rid of the controversy results obtained from different groups [9].

Table 1 and Figure 2 show the model studies in the country and the seropositivity rate in patients. As it can be seen, no studies have been performed in the eastern and central parts of Iran and the reason is the low prevalence of the disease especially in southeastern areas of the country. The eastern and central parts of Iran do not have enough pasture. That is why it is not a good place for animal husbandry, and consequently there are not enough intermediate hosts for this kind of helminthes. Furthermore, the parasite egg is sensitive to high temperature and low humidity. So, on one hand lack of intermediate host for this worm and on the other hand existing industrial abattoir make the life cycle of such helminthes unfinished because the infected meat of intermediate hosts is not available for the final host. Based on the mentioned reasons we have less infected people with HCE. That is why we have a small number of infected people who do surgery to remove cysts. This issue makes the seroepidemiological study difficult because of the lack of samples [21]. On the other hand, the highest prevalence rate of this parasite belongs to the western parts of the country especially in the provinces of Lorestan, Kohgiluyeh and Boyer-Ahmad, Khuzestan, Fars, and Ardabil which have a mild climate and more rainfall and humidity. This fact consequently increases the survival rate of the eggs and the transmission cycle of the parasite. Therefore, the different climate conditions of the country have an effective role in the infection prevalence rate. On the other hand, due to the good weather conditions, the rate of livestock raising and grazing is very high in these areas which can cause parasite infection in the intermediate hosts and eventually in the final hosts. It must be noted that the stray dogs which are infected with the parasite play a very big role in spreading the disease and increasing the prevalence rate in these areas [11].

On average, the hydatidosis rate in stray dogs is 5$94 \%$ in different regions of Iran [8] and the median rate of infection in the stray dogs is $20 \%$ in the western areas [9] which shows a high rate of infection in the final hosts. One more thing to be kept in our mind is about cultural and religious issues existing in Iran. Here in Iran in the rural parts, most of the people have dogs and these dogs are vaccinated and cannot consider them as a source of infections [3]. These infected stray dogs can easily spread large numbers of parasite eggs on the agricultural fields where vegetables grow. This is considered as one of the main factors in increasing the prevalence of the disease in intermediate hosts including humans in these regions. Also the human behavior in contact with dogs plays an important role in the transmission of infection in humans. This behavior is closely related to peoples' cultural and economic conditions. Because people who keep dogs in these areas are more sensitive to the 
dogs' infections, these people are also inferior to other people in terms of educational and economic conditions [8].

This study identified that the prevalence of HCE infection was usually higher in rural inhabitants rather than in urban ones $[16,19,22-26]$; also most of the studies showed that females were the main subject of HCE [9, 20, 22, 25, 27-31]. One evidence of this result is that most farmers and housewives that come in contact with infection source are females that live in rural areas [8]. In addition, the presence of an unlimited dog population in rural communities contributes to the exposure to E. granulosus and high seroprevalence rate. Slaughtering practices (noncontrolled family slaughtering in rural areas versus more publicly or privately organized and controlled slaughtering in cities, especially Tehran) and permanent presence of intermediate hosts (sheep) to maintain the cycle are important too [5].

The results of our investigation showed that prevalence of HCE is high among subjects in the middle-aged people in most of the studies. They reported the age range of 10-19 as the highest infected age group in Zanjan [29], 20-40 age range in Kurdistan [32], 60-69, 60-80, and 60-90 age range in Isfahan, Hamadan, and Ardabil [18, 26, 33], 20-29 years old in Khorramabad [16], 30-39 years old in Yasuj [24], and 2939, 40-49, and 30-60 years old in Kerman, Arak, Golestan, and Qom, respectively [19, 34-36].

These heterogeneity differences seen in the studies performed in different regions of the country are due to the classification of different age groups, the difference in the type of the studied people, and the geographical location of each study. Also long patent period of this disease is one of the problems [18]. The issue of age groups also includes the population's access to care: if diagnosis is made rather early because of easy availability of US examination and if young people are operated on because of the proximity of well-equipped hospitals and of surgeons ready to work (and earn their living!), there will be higher prevalence in older age groups. As different studies show different results and with most cases being diagnosed many years after their infection, the detection of true age group in the context of infection with HCE is more difficult. Mass screening has well shown that the majority of cysts will remain asymptomatic and even spontaneously degenerate in the majority of subjects; serology does not distinguish between rapidly progressing cases and stable or even aborted cases. Several publications support this [11].

4.1. Strengths and Weaknesses of Study Designs. These studies demonstrate the importance of serologic HCE in various groups of people in Iran. The control of the disease in Iran where most dogs are stray and are infected with adult worms all around the cities is difficult. Therefore, public education that highlights the importance of washed vegetables and inhabitation of exposure to the source of infection could reduce the transmission of the parasite and the consequences of infection in humans $[6,13]$.

In general, performing such systematic studies on the rate of the human hydatid cysts seroepidemiology in different parts of the world especially in parasite endemic countries like Iran which is located in the Middle East can show the general trend of the disease in its different parts. Studying the general pattern of the disease in different regions can help a lot in planning health and disease prevention programs. Considering the very high economic losses in terms of mutilating the infected organs of the domestic animals as well as the various surgeries performed on the infected humans existing in the country, these control programs can decrease the general trend of the disease.

\section{Conclusion}

HCE is highly prevalent in Iran and could be a cause of considerable health problems in the country. Educational programs, serological screening, and the continuation of the treatment of the patients when possible could help reduce the national impacts of the disease. Further studies are needed to describe the exact epidemiology of the disease at a national level in other parts of Iran.

\section{Competing Interests}

The authors declare that there are no competing interests.

\section{References}

[1] Z. Pawlowski and D. Vuitton, "Echinococcosis in humans: clinical aspects, diagnpsis and treatment," in WHO/OIE Manual on Echinococcosis in Humans and Animals: A Public Health Problem of Global Concern, J. Eckert, M. A. Gemmel, F.-X. Meslin, and Z. S. Pawłowski, Eds., pp. 20-66, World Health Organization and World Organization for Animal Health, Paris, France, 2001.

[2] W. Zhang, H. Wen, J. Li, R. Lin, and D. P. McManus, "Immunology and immunodiagnosis of cystic echinococcosis: an update," Clinical and Developmental Immunology, vol. 2012, Article ID 101895, 10 pages, 2012.

[3] M. Fasihi Harandi, C. M. Budke, and S. Rostami, "The monetary burden of cystic echinococcosis in Iran," PLoS Neglected Tropical Diseases, vol. 6, no. 11, Article ID e1915, 2012.

[4] P. R. Torgerson and P. Deplazes, "Echinococcosis: diagnosis and diagnostic interpretation in population studies," Trends in Parasitology, vol. 25, no. 4, pp. 164-170, 2009.

[5] P. Moro and P. M. Schantz, "Echinococcosis: a review," International Journal of Infectious Diseases, vol. 13, no. 2, pp. 125-133, 2009.

[6] S. M. Sadjjadi, "Present situation of echinococcosis in the Middle East and Arabic North Africa," Parasitology International, vol. 55, supplement, pp. S197-S202, 2006.

[7] M. B. Rokni, "The present status of human helminthic diseases in Iran," Annals of Tropical Medicine and Parasitology, vol. 102, no. 4, pp. 283-295, 2008.

[8] M. B. Rokni, "Echinococcosis/hydatidosis in Iran," Iranian Journal of Parasitology, vol. 4, pp. 1-16, 2009.

[9] A. Dalimi, G. Motamedi, M. Hosseini et al., "Echinococcosis/hydatidosis in western Iran," Veterinary Parasitology, vol. 105, no. 2, pp. 161-171, 2002.

[10] M. F. Harandi, R. P. Hobbs, P. J. Adams, I. Mobedi, U. M. Morgan-Ryan, and R. C. A. Thompson, "Molecular and morphological characterization of Echinococcus granulosus of human and animal origin in Iran," Parasitology, vol. 125, no. 4, pp. 367-373, 2002. 
[11] B. Sarkari and Z. Rezaei, "Immunodiagnosis of human hydatid disease: where do we stand?" World Journal of Methodology, vol. 5, no. 4, pp. 185-195, 2015.

[12] M. B. Rokni and B. Aminian, "Evaluation of the Enzymelinked Immuno-electro Transfer Blot (EITB) technique using hydatid cyst antigens $\mathrm{B} / 5$ and total IgG antibodies in laboratory diagnosis of human hydatidosis," Pakistan Journal of Medical Sciences, vol. 22, no. 2, pp. 127-131, 2006.

[13] S. M. Sadjjadi, S. Ardehali, B. Noman-Pour, V. Kumar, and A. Izadpanah, "Diagnosis of cystic echinococcosis: ultrasound imaging or countercurrent immunoelectrophoresis?" Eastern Mediterranean Health Journal, vol. 7, no. 6, pp. 907-911, 2001.

[14] H. Z. Hezarjaribi, M. Fakhar, A. Shokri, S. Hosseini Teshnizi, A. Sadough, and M. Taghavi, "Trichomonas vaginalis infection among Iranian general population of women: a systematic review and meta-analysis," Parasitology Research, vol. 114, no. 4, pp. 1291-1300, 2015.

[15] M. T. Rahimi, A. Daryani, S. Sarvi et al., "Cats and Toxoplasma gondii: a systematic review and meta-analysis in Iran," Onderstepoort Journal of Veterinary Research, vol. 82, no. 1, article 823, pp. 1-10, 2015.

[16] M. Zibaei, A. Azargoon, M. Ataie-Khorasgani, K. Ghanadi, and S. M. Sadjjadi, "The serological study of cystic echinococcosis and assessment of surgical cases during 5 years (2007-2011) in Khorram Abad, Iran," Nigerian Journal of Clinical Practice, vol. 16, no. 2, pp. 221-225, 2013.

[17] G. L. B. Farrokhzad, M. Nariman, and M. R. Nazari Poya, "Investigation of the prevalence of hydatid cysts in rural areas shemiranat of Tehran and reviews of IFA test," Research in Medicine, vol. 30, no. 3, pp. 241-244, 2004.

[18] M. Arbabi, J. Masoud, A. Dalimi Asl, and M. Sajadi, "Seroepidemiologic prevalence of Hydatid cyst in Hamadan," Feyz, vol. 2, no. 2, pp. 43-50, 1998.

[19] M. Asgari, M. Mohebali, E. B. Kia et al., "Seroepidemiology of human hydatidosis using AgB-ELISA test in Arak, central Iran," Iranian Journal of Public Health, vol. 42, no. 4, pp. 391-396, 2013.

[20] A. Rafiei, A. Hemadi, S. Maraghi, B. Kaikhaei, and P. S. Craig, "Human cystic echinococcosis in nomads of south-west Islamic Republic of Iran," Eastern Mediterranean Health Journal, vol. 13, no. 1, pp. 41-48, 2007.

[21] M. Saberi-Firouzi, F. Kaffashian, E. Hayati et al., "Prevalence of hydatidosis in nomadic tribes of southern Iran," Medical Journal of the Islamic Republic of Iran, vol. 12, no. 2, pp. 113-118, 1998.

[22] A. Aflaki, F. Ghaffarifar, and A. Dalimi Asl, "Seroepidemiological survey of hydatidosis by Dot- ELISA in Ilam province," Modarres Journal of Medical Sciences, vol. 8, pp. 1-6, 2005.

[23] M. Hadadian, F. Ghaffarifar, A. Dalimi Asl, and S. Roudbar Mohammadi, "Seroepidemiological survey of hydatid cyst by ELISA in Kordestan province," Modares Journal of Medical Sciences: Pathobiology, vol. 10, pp. 13-18, 2008.

[24] B. Sarkari, S. M. Sadjjadi, M. M. Beheshtian, M. Aghaee, and F. Sedaghat, "Human cystic echinococcosis in Yasuj district in Southwest of Iran: an epidemiological study of seroprevalence and surgical cases over a ten-year period," Zoonoses and Public Health, vol. 57, no. 2, pp. 146-150, 2010.

[25] S. Kavous, A. Kazemi, and S. Jelodari, "Seroepidemiology of human hydatid cyst in jahrom," Journal of Jahrom Uiversity of Medical Sciences, vol. 8, no. 3, pp. 18-24, 2010.

[26] Z. Heidari, M. Mohebali, Z. Zarei et al., "Seroepidemiological study of human hydatidosis in Meshkinshahr district, Ardabil province, Iran," Iranian Journal of Parasitology, vol. 6, no. 3, pp. 19-25, 2011.
[27] M. Sedaghat Gohar, J. Massoud, M. Rokni, and E. Kia, "Seroepidemiologic survey of human hydatidosis in Shahriar region," Journal of Kerman University of Medical Sciences, vol. 1, pp. 4449, 2001.

[28] H. Yousefi Darani, M. Avijgan, K. Karimi, K. Manouchehri, and J. Masood, "Seroepidemiology of hydatid cyst in Chaharmahal va Bakhtiari Province," Iranian Journal of Public Health, vol. 32, no. 2, pp. 31-33, 2003.

[29] A. Haniloo, H. Badali, and A. Esmaeil Zadeh, "Seroepidemiological study of Hydatidosis in Zanjan, Islam-Abad, 2002," Journal of Zanjan University of Medical Sciences \& Health Services, vol. 12, pp. 41-46, 2002.

[30] H. Mirzanejadasl, M. F. Harandi, and P. Deplazes, "Serological survey of human cystic echinococcosis with ELISA method and CHF Ag, in moghan plain, Ardabil Province, Iran," Research Journal of Biological Sciences, vol. 3, no. 1, pp. 64-67, 2008.

[31] S. S. Moazezi, M. Fasihi Harandi, M. Saba, H. Kamyabi, and F. Sheikhzadeh, "Sonographic and serological survey of hydatid disease in rural regions of Shahdad and Chatroud, Kerman province, 2006-2007," Journal of Kerman University of Medical Sciences, vol. 16, no. 1, pp. 25-34, 2009.

[32] S. A. Hosseini and J. Masood, Seroepidemiological study of hydati-dosis in Divandarreh, Kurdistan [M.S. thesis], Medical Parasitology, School of Public Health, Tehran University of Medical Sciences, Tehran, Iran, 1997.

[33] P. Ilbeigi, M. Mohebali, E. B. Kia et al., "Seroepidemiology of human hydatidosis using AgB-ELISA test in Isfahan City and Suburb Areas, Isfahan Province, Central Iran," Iranian Journal of Public Health, vol. 44, no. 9, pp. 1219-1224, 2015.

[34] M. Baharsefat, J. Massoud, I. Mobedi, A. Farahnak, and M. Rokni, "Seroepidemiology of cystic echinococcosis in referred patients to health centers in Golestan Province using ELISA and IFA," Iranian Journal of Parasitology, vol. 2, pp. 20-24, 2007.

[35] M. F. Harandi, S. S. Moazezi, M. Saba et al., "Sonographical and serological survey of human cystic echinococcosis and analysis of risk factors associated with seroconversion in rural communities of Kerman, Iran," Zoonoses and Public Health, vol. 58 , no. 8, pp. 582-588, 2011.

[36] A. Rakhshanpour, M. Fasihi Harandi, S. S. Moazezi et al., "Seroprevalence of human hydatidosis using ELISA method in Qom province, central Iran," Iranian Journal of Parasitology, vol. 7, no. 3, pp. 10-15, 2012.

[37] R. Jamali, B. Naghili, and Sh. Mozafari, "Seroepidemiological study of Hydatid cyst prevalence in Uromieh villagers," Medical Journal of Tabriz University of Medical Sciences \& Health Service, vol. 29, no. 27, pp. 23-30, 1995.

[38] H. Mohamadi, Seroepidemiological study of hydatidosis in man in Varamin area south of Tehran [M.S. dissertation], Tehran University of Medical Sciences, Irantion SoPH, Tehran, Iran, 1998.

[39] M. R. Zariffard, N. Abshar, M. A. Akhavizadegan, and G. R. Motamedi, "Seroepidemiological survey of human hydatidosis in western parts of Iran," Archives of Razi Institute, vol. 50, pp. 71-75, 1999.

[40] M. R. Nilfroshan, A. Deylami, and H. Niazi, "Epidmiology of Hydatid cyst in Fardin dirstrict," Pajouhesh Va Sazandgi, vol. 36, pp. 80-83, 1998.

[41] Z. Amiri, Prevalence of hydatid cyst in Kermanshah using IFA and ELISA method in the year 2001 [M.S. thesis], Medical Parasitology, School of Public Health, Tehran University of Medical Sciences, Tehran, Iran, 2001. 
[42] A. Rafiei, P. S. Craig, and S. A. Maraghi, "Seroepidemiological servey of human cysitic ecinicoccnsis in Iran," in Proceedings of the 20th International Congress of Hydatidology, vol. 193, Kusadasi, Turkey, June 2001.

[43] L. Akhlaghi, J. Massoud, and A. Housaini, "Observation on hydatid cyst infection in Kordestan province (West of Iran) using epidemiological and seroepidemiological criteria," Iranian Journal of Public Health, vol. 34, no. 4, pp. 73-75, 2005.

[44] L. Akhlaghi, H. Ourmazdi, S. H. Sarvi et al., "Using Dot-ELISA method to study the prevalence of human hydatidosis in people referred to blood transfusion center in Tehran, 2005-2006," Journal of Iran University of Medical Sceinces, vol. 16, no. 67, pp. 52-58, 2009.

[45] N. Esmaeili and M. Arbabi, "Seroepidemiology of hydatidosis among adult human at Kashan region, Iran in 2008," Journal of Kashan University of Medical Sciences, vol. 13, no. 4, pp. 321-326, 2010.

[46] M. A. Dadkhah, M. Yeganehzad, and B. Nadery, "Survey on hydatid cyst infestation in Sarab city (Northwest of Iran) using epidemiological and seroepidemiological," Journal of Animal and Veterinary Advances, vol. 10, no. 16, pp. 2099-2101, 2011.

[47] Y. Garedaghi and S. R. Bahavarnia, "Seroepidemiology of human hydatidosis by ELISA method in East-Azarbaijan province in Iran in year 2009," Iranian Journal of Epidemiology, vol. 7, no. 2, pp. 25-29, 2011.

[48] R. Shahrokhabadi, E. Rahimi, and R. Poursahebi, "Seroepidemiological study of human hydatidosis in Rafsanjan, Kerman," Zahedan Journal of Research in Medical Sciences, vol. 16, no. 4, p. 46, 2014. 

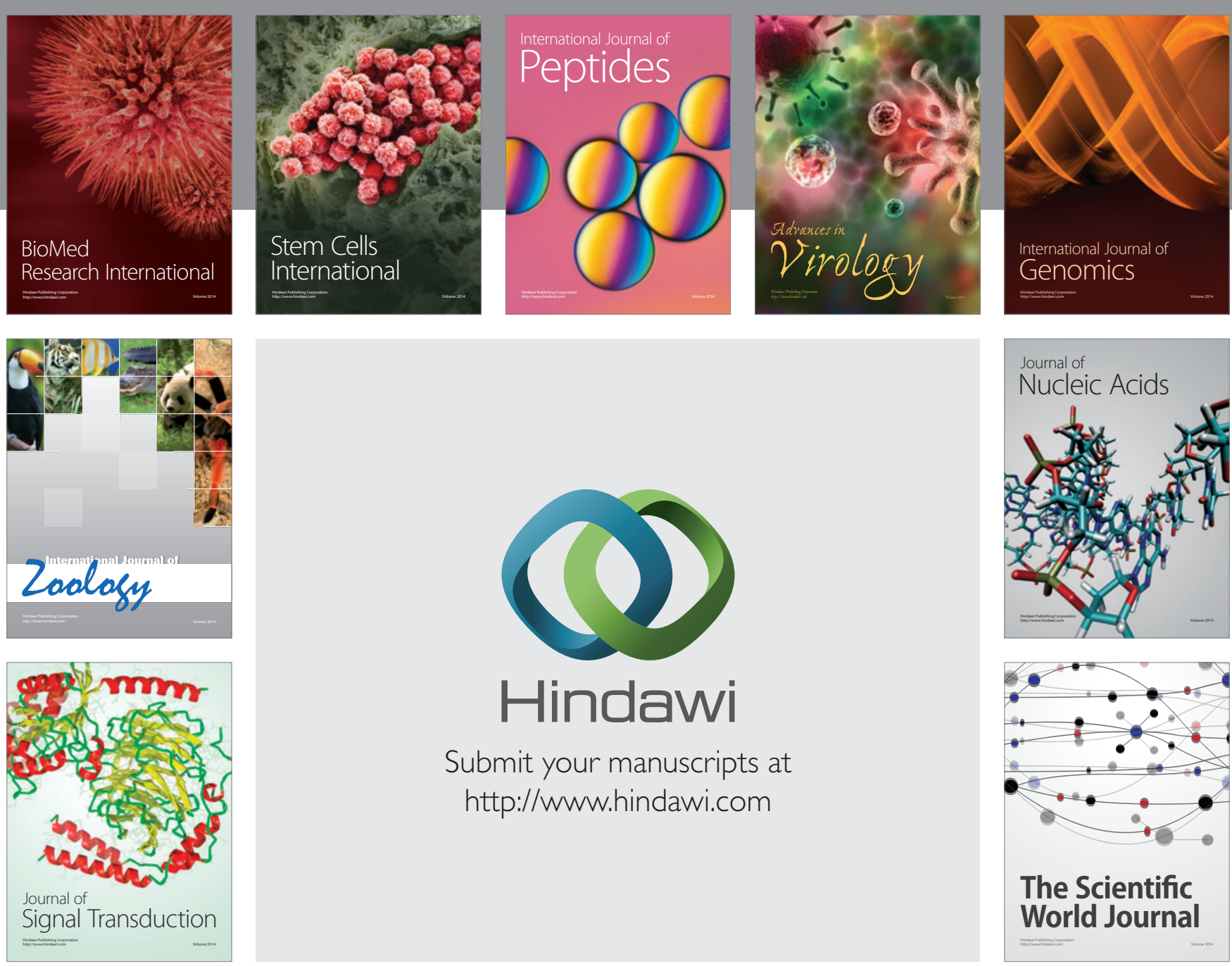

Submit your manuscripts at

http://www.hindawi.com
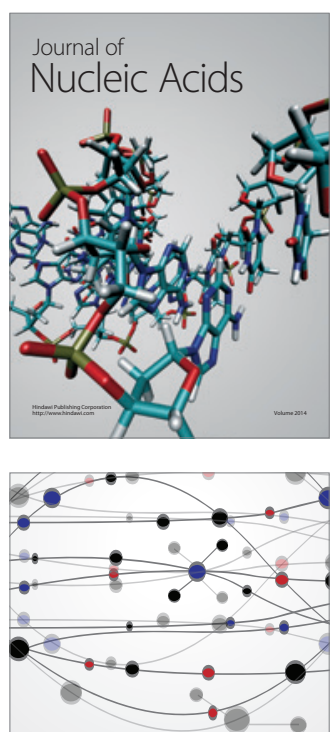

The Scientific World Journal
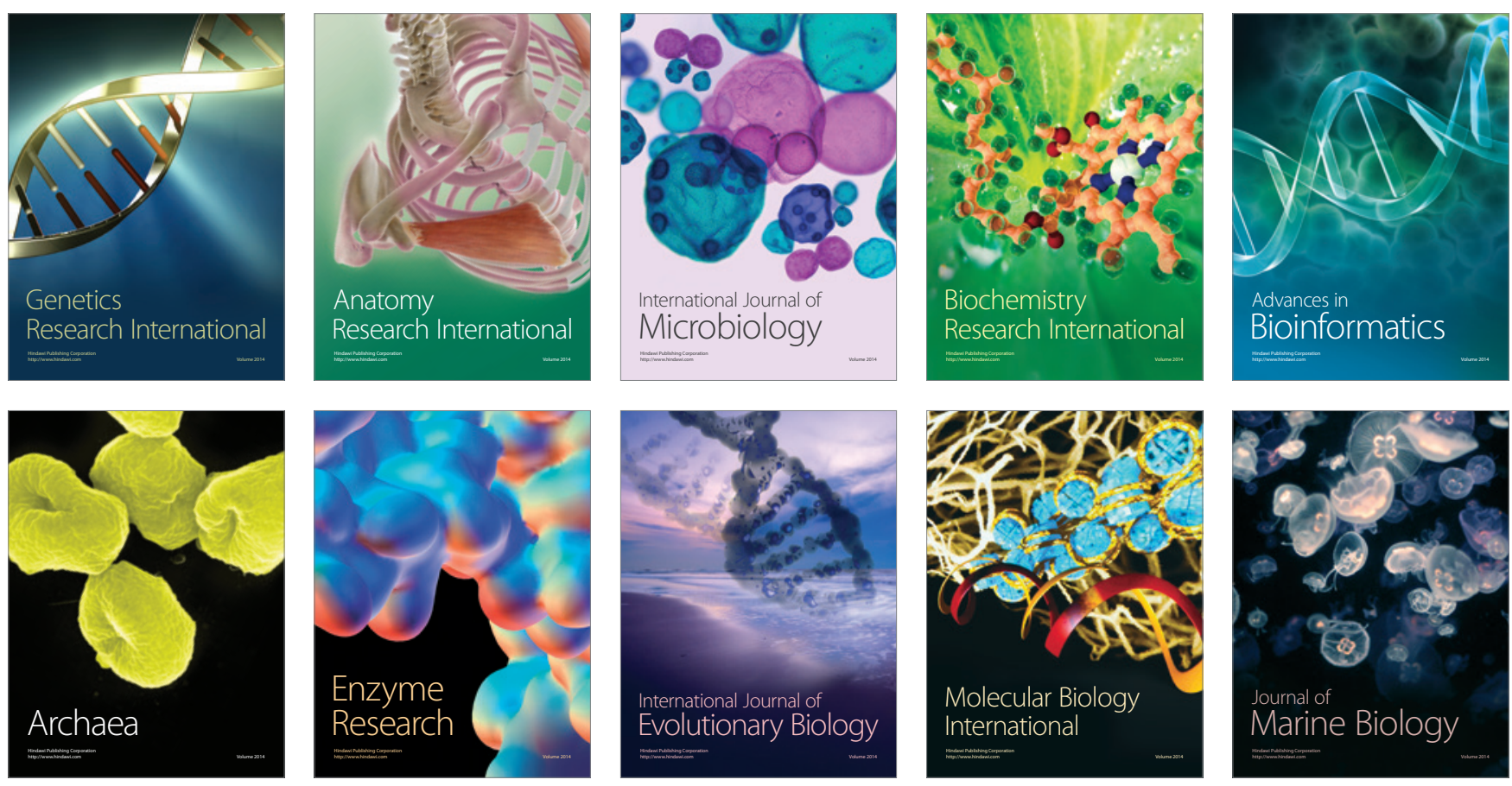